REPORT NO.

UCD/CGM-01/01
CENTER FOR GEOTECHNICAL MODELING

\section{TENSOR VISUALIZATIONS IN COMPUTATIONAL GEOMECHANICS}

BY

B. JEREMIC

G. SCHEUERMANN

J. FREY

Z. YANG

B. HAMMAN

K.I. JOY

H. HAGGEN

DEPARTMENT OF CIVIL \& ENVIRONMENTAL ENGINEERING COLLEGE OF ENGINEERING UNIVERSITY OF CALIFORNIA AT DAVIS 
INTERNATIONAL JOURNAL FOR NUMERICAL AND ANALYTICAL METHODS IN GEOMECHANICS Int. J. Numer. Anal. Meth. Geomech. 2001; 01:1-6 Prepared using nagauth.cls [Version: 2000/03/22 v1.0]

\title{
Tensor Visualizations in Computational Geomechanics
}

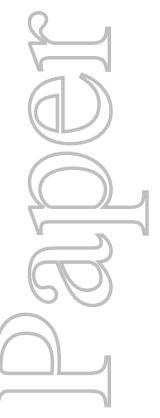

\author{
Boris Jeremic ${ }^{1 *}$, Gerik Scheuermann ${ }^{2},{ }^{3}$, Jan Frey ${ }^{3}$, Zhaohui Yang ${ }^{1}$, Bernd \\ Hamann $^{2}$, Kenneth I. Joy ${ }^{2}$, Hans Hagen ${ }^{3}$ \\ ${ }^{1}$ Department of Civil and Environmental Engineering, University of California, Davis, CA 95616-5270, \\ U.S.A. \\ ${ }^{2}$ Center for Image Processing and Integrated Computing (CIPIC), Department of Computer Science, \\ University of California, Davis, CA 95616-8562, U.S.A. \\ 3 Computer Science Department, University of Kaiserslautern, P.O. Box 3049, D-67653 Kaiserslautern, \\ Germany
}

submitted for publication in the International Journal for Numerical and Analytical Methods in Geomechanics, May 2001.

\footnotetext{
${ }^{*}$ Correspondence to: Boris Jeremić, Department of Civil and Environmental Engineering, University of California, One Shields Ave., Davis, CA 95616-5270, U.S.A. jeremic@ucdavis .edu

Contract/grant sponsor: ; contract/grant number:
}

Copyright (C) 2001 John Wiley \& Sons, Ltd. 
We present a novel technique for visualizing tensors in three dimensional (3D) space. Of particular interest is the visualization of stress tensors resulting from 3D numerical simulations in computational geomechanics. To this end we present three different approaches to visualizing tensors in $3 \mathrm{D}$ space, namely hedgehogs, hyperstreamlines and hyperstreamsurfaces. We also present a number of examples related to stress distributions in 3D solids subjected to single and load couples. In addition, we present stress visualizations resulting from single-pile and pile-group computations.

Copyright (C) 2001 John Wiley \& Sons, Ltd.

KEY WORDS: Tensor Visualization, Computational Geomechanics

\section{INTRODUCTION}

Over the last couple of years, large--scale 3D finite element models were developed in computational geomechanics (cf. Jeremić et al. [10], Bao et al. [1, 2]) Despite significant efforts to develop better models to computationally simulate a number of interesting problems in geomechanics, post-processing and data analysis are still a big burden. As a consequence, simulation results are usually presented in traditional $2 \mathrm{D}$ format with two axes representing interaction of two scalar variables. Moreover, the scalar variables are usually picked up at certain points in space (or time). The analyst needs to have considerable experience to pick up spatial coordinate and two variables to be followed. This approach is suitable only for simple $1 \mathrm{D}$ and some $2 \mathrm{D}$ problems. In analyzing results of $3 \mathrm{D}$ computations, the analyst is faced with

0
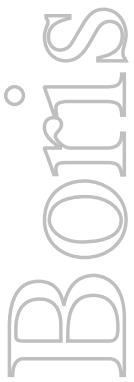

a large number of output data points. Each data point can have multiple associated variables Copyright (C) 2001 John Wiley \& Sons, Ltd. Int. J. Numer. Anal. Meth. Geomech. 2001; 01:1-6 
(scalar or tensorial). In addition, post-processing of results from dynamic computations tends to introduce new, derived data sets that can be even larger that the original simulations output data sets. The major problem is to "sort out" the output data in an economical way and present it intuitively to the analyst.

One possible approach is to use advanced computer graphics and visualizations tools for the task of post-processing large geomechanics data sets. In addition to presenting output results in a graphical way (in terms of stress hedgehogs, for example) we have developed methods new methods to extract more information from a stress tensor field and present it in an intuitive way. This new methods are based on the concepts of so called hyperstreamlines and hyperstreamsurfaces (Delmarcelle and Hesselink [3, 4], Hagen et al. [8]).

In addition, new visualization hardware can be used to improve the quality and experience of the visualization process. We mention the Immersive WorkBench from FakeSpace Inc. [6] available at the Center for Image Processing and Integrating Computing (CIPIC) at UC Davis. The Immersive WorkBench makes it possible to visualize objects in 3D space directly, using stereoscopic rendering technology. Moreover, when combined with the data glove (part of the WorkBench's set-up) it creates a complete virtual environment in which the analyst can manipulate 3D objects with his/her own hands.

We note note that, although the visualization tools were designed for post-processing stress data, the methods and implementations developed are highly general and can be used for any $3 \mathrm{D}$ tensor field.

The paper is organized as follows: In Section 2, we give brief introduction to tensor fields and then focus on the stress tensor. Section 3 describes various implementation details, in particular, it discusses manipulations of the provided data sets in order to obtain Copyright (C) 2001 John Wiley \& Sons, Ltd. Int. J. Numer. Anal. Meth. Geomech. 2001; 01:1-6 Prepared using nagauth.cls 
"optimal tensor fields" for visualization. In Section 4, we present three methods (hedgehogs, hyperstreamlines and hyperstreamsurfaces) for visualizing stress tensor fields. We present a number of illustrative examples. In section 5, we present conclusions and directions for future work.

\section{BASICS OF TENSOR FIELDS}

We briefly review definitions and basic properties of tensors. We follow notation of Green and Zerna [7] and of Hjelmstad [9]. Let us consider three independent variables $\theta^{i}$ with differentials $d \theta^{i}$. The variables $\theta^{i}$ are transformed into a set of new variables $\bar{\theta}^{i}$ by an arbitrary single-valued function of the form

$$
\bar{\theta}^{i}=\bar{\theta}^{i}\left(\theta^{1}, \theta^{2}, \theta^{3}\right)
$$

We assume that the arbitrary function has derivatives up to the order required. We also assume that the function is invertible, i.e.,

$$
\theta^{i}=\theta^{i}\left(\bar{\theta}^{1}, \bar{\theta}^{2}, \bar{\theta}^{3}\right)
$$

where the functions $\theta^{i}$ are also single-valued.

The transformation of differentials is given by the equations

$$
d \bar{\theta}^{i}=\bar{c}_{j}^{i} d \theta^{j} \quad \text { and } \quad d \theta^{i}=c_{j}^{i} d \bar{\theta}^{j}
$$

where we define

$$
\bar{c}_{j}^{i}=\frac{\partial \bar{\theta}^{i}}{\partial \theta^{j}} \quad \text { and } \quad c_{j}^{i}=\frac{\partial \theta^{i}}{\partial \bar{\theta}^{j}}
$$

The functions in Eq. 4 are related by the equation

$$
\bar{c}_{k}^{i} c_{j}^{k}=c_{k}^{i} \bar{c}_{j}^{k}=\delta_{j}^{i}
$$

Copyright (C) 2001 John Wiley \& Sons, Ltd.

Int. J. Numer. Anal. Meth. Geomech. 2001; 01:1-6

Prepared using nagauth.cls 
where $\delta_{j}^{i}$ is the Kronecker delta. Eq. 5 can be used to calculate the values of $\bar{c}_{j}^{i}$ when the values of $c_{j}^{i}$ are known. The opposite relationship also holds, provided that the functional determinant $c=\left\|\bar{c}_{j}^{i}\right\| \neq 0$, which holds because of the assumption of invertibility (Eq. 2).

Consider a system $A$ of functions with components defined in the general set of variables $\theta^{i}$ and being functions of $\left(\theta^{1}, \theta^{2}, \theta^{3}\right)$. We call the system of functions $A$ a tensor if the variables $\theta^{i}$ can be changed to $\bar{\theta}^{i}$ by Eq. 1 and if we can define new components of $T$ in the general variables $\bar{\theta}^{i}$, being functions of $\left(\bar{\theta}^{1}, \bar{\theta}^{2}, \bar{\theta}^{3}\right)$ and if the components of $T$ in the two sets of variables are related by certain rules.

A system of order two may be defined by nine components $A_{i j}$ in the variables $\theta^{i}$ and nine components $\bar{\theta}^{i}$ in the variables $\bar{\theta}^{i}$. If

$$
\bar{A}_{i j}=c_{i}^{m} c_{j}^{n} A_{m n}
$$

the functions $A_{i j}\left(\theta^{1}, \theta^{2}, \theta^{3}\right)$ and $\bar{A}_{i j}\left(\bar{\theta}^{1}, \bar{\theta}^{2}, \bar{\theta}^{3}\right)$ are the components in their respective variables of a covariant tensor of order two. Tensor $A_{i j}$ is said to be symmetric if

$$
A_{i j}=A_{j i}
$$

Consider a body $\boldsymbol{B}$ (Figure 1) subjected to surface forces, and an interior surface element of area $\Delta \boldsymbol{A}$ with normal $\boldsymbol{n}$ and resultant force $\Delta \boldsymbol{F}$, see Hjelmstad [9] and Sukumar and Rashid [11]. The traction vector can then be defined as the limit of the ratio of the vectorial force to the area

$$
\boldsymbol{t}=\lim _{\Delta \boldsymbol{A} \rightarrow 0} \frac{\Delta \boldsymbol{F}}{\Delta A}
$$

The Cauchy stress tensor $\boldsymbol{\sigma}\left(\sigma_{i j}\right)$ is defined as a tensorial quantity that, when acted on by the unit normal $\boldsymbol{n}\left(n_{i}\right)$, results in the traction vector $\boldsymbol{t}\left(t_{j}\right)$ :

$$
\boldsymbol{t}=\boldsymbol{n} \boldsymbol{\sigma}
$$




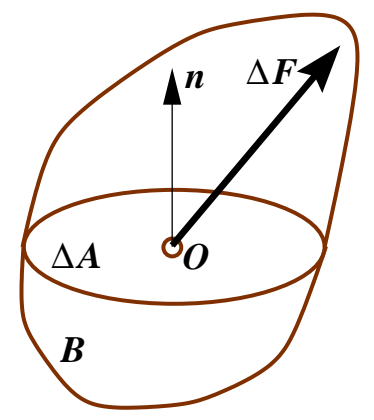

Figure 1. Strained body $\boldsymbol{B}$ with a planar cross section $\Delta A$, unit normal $\boldsymbol{n}$ and a force $\Delta \boldsymbol{F}$.

$$
t_{i}=n_{j} \sigma_{i j}
$$

The components of the Cauchy stress tensor are shown in Figure 2. It can be shown that

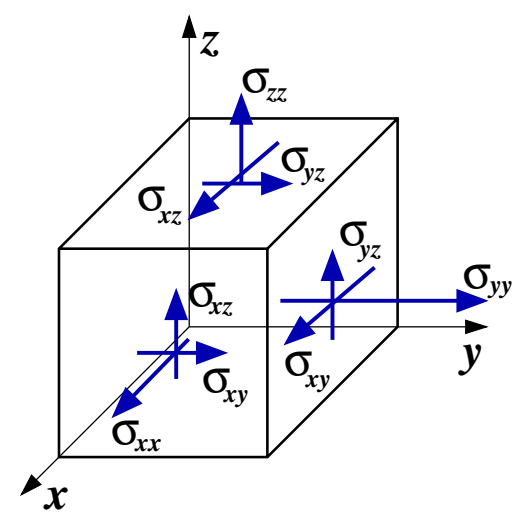

Figure 2. Components of the Cauchy stress tensor $\sigma_{i j}$ (components shown on three visible planes only).

the Cauchy stress tensor is symmetric, i.e. $\sigma_{i j}=\sigma_{j i}$.

It is of interest to determine on which planes the traction vector $t_{i}$ and plane normal $n_{j}$ are aligned as for that plane the shear stress is zero. In other words, we use Eq. 10 and seek to determine planes with normals $n_{i}$ where $t_{i}=\sigma n_{i}$ is satisfied. Using the above equations, we Copyright (C) 2001 John Wiley \& Sons, Ltd.

Int. J. Numer. Anal. Meth. Geomech. 2001; 01:1-6 Prepared using nagauth.cls 
have

$$
n_{j} \sigma_{j i}=\sigma n_{i}
$$

leading to the equation

$$
\left(\sigma_{i j}-\sigma \delta_{i j}\right) n_{j}=0
$$

and hence it follows that

$$
\operatorname{det}\left(\sigma_{i j}-\sigma \delta_{i j}\right)=0
$$

for the eigenvalue problem to have a non-trivial solution. The values $\sigma$ are the eigenvalues (principal stresses) $\sigma_{I}, I=1,2,3$ having associated eigenvectors (principal directions) $n_{I}$ that are normal to the principal planes.

\section{DATA STRUCTURES AND CONVERSION}

Finite element models used in geomechanics simulations can feature a number of different mesh arrangements. For simpler models, used in testing the quality of implementation or error propagations, meshes are usually fairly regular in shape. For modeling complex realistic problems, finite element meshes become irregular. For example, Figures $3(\mathrm{a}-\mathrm{c})$ show test problems used in this work. In these examples, the domain is rectangular in shape with different loads applied on top face. On the other hand, Figures $3(\mathrm{~d}-\mathrm{e})$ show solid models of realistic pile foundations. It should be noted that the piles shown in Figures $3(\mathrm{~d}-\mathrm{e})$ are embedded in soil, and the complete models (for both a single pile and pile groups) include large volume of soils surrounding the pile foundations.

A static and dynamic finite element simulation of a 3D problem results in large output, typically several very large data files. For some of the examples shown later, the output files Copyright (C) 2001 John Wiley \& Sons, Ltd.

Int. J. Numer. Anal. Meth. Geomech. 2001; 01:1-6 Prepared using nagauth.cls 

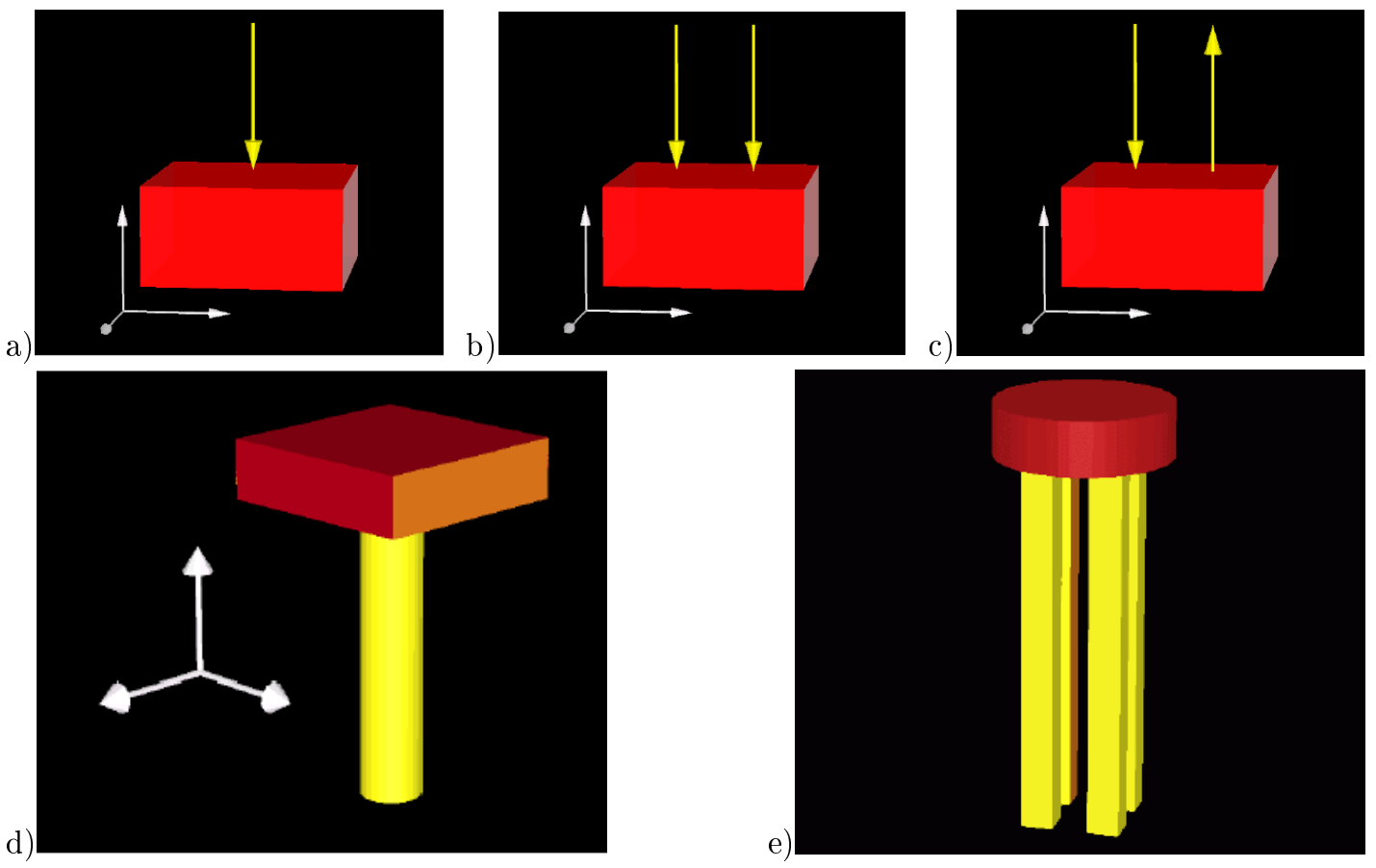

Figure 3. Rectangular domains with different loads applied on top face, (a) point load, (b) double point load, (c) load couple. Solid models of realistic pile foundations, (d) single pile, and (e) four pile group.

contain only nodal (vertex) displacements and stress tensors associated with Gauss points (used for numerical integration), file size typically exceeds 1 Gigabyte of data.

An important issue that must be resolved before the data can be visualized is the location of data points. Standard-displacement-based finite element methods usually provide displacement data at nodal points (mesh vertices) while the constitutive data (e.g., the dependent stress and strain tensors, and/or material model data) are provided at the Gauss points within each finite element. For example, Figure 4(a) is a top view of the single-pile mesh used in our examples. It is obvious that the availability of displacement data at nodal points and constitutive data at Gauss points can lead to potential problems for the creation Copyright (C) 2001 John Wiley \& Sons, Ltd.

Int. J. Numer. Anal. Meth. Geomech. 2001; 01:1-6 Prepared using nagauth.cls 
of a smooth field obtained by interpolation.

One of the first ideas we explored was to extrapolate Gauss point data to nodal points and then perform averaging, see Zienkiewicz and Taylor [12]. Figure 4(b) shows how Gauss-point data (pointed to by red arrows) was extrapolated to nodal points (indicated by green arrows). However, since the stress field resulting from a finite element simulation, is not continuous across element boundaries, this extrapolation approach created, in some cases, non-physical stress distributions. Moreover, it is quite difficult to implement this type of data conversion for non-regular meshes. There does not seem to exist a simple method to extrapolate Gauss-point data for a non-regular meshes.

Instead of extrapolating Gauss-point data to nodal points we can use the concepts of a Delaunay triangulation, see Hagen et al. [8], to perform data conversion. The advantages of performing a Delaunay-based conversion are numerous. We mention just a few advantages:

- The conversion results in tetrahedral cells only.

- The conversion is independent of the data set.

- The conversion is independent of the number of Gauss points in an element.

On the downside, this type of conversion results in a rather large increase in the number of cells. Considering the simple test mesh used for the examples in Figure 4(a), with 600 elements and 4800 Gauss points (eight Gauss points per element), the Delaunay conversion will yield 26820 tetrahedral cells. Figure 4(c) shows a top view of the tetrahedrazation of the initial mesh for a single pile, while Figure 4(d) shows the tetrahedrazation for the Gauss points of

O

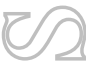

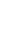
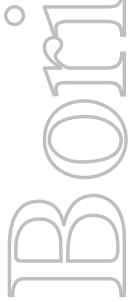

Int. J. Numer. Anal. Meth. Geomech. 2001; 01:1-6 


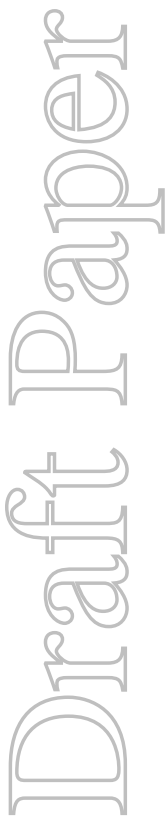

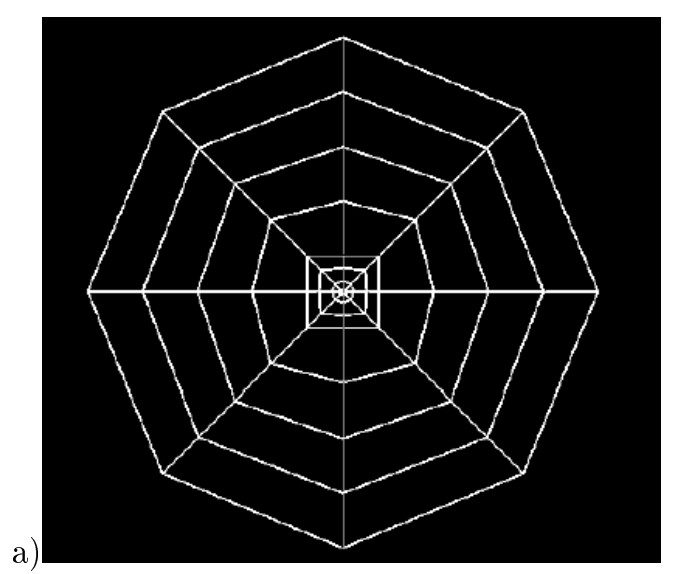

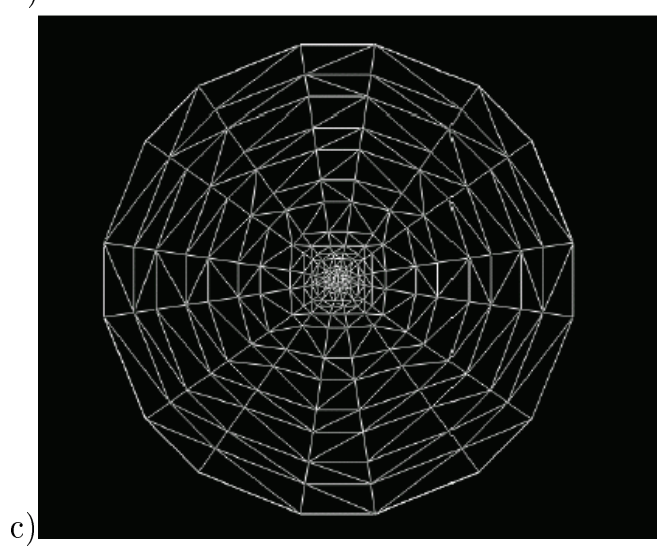

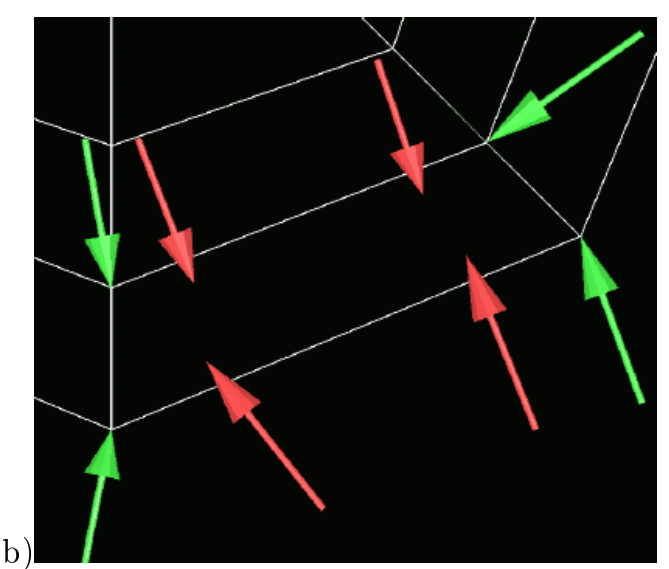

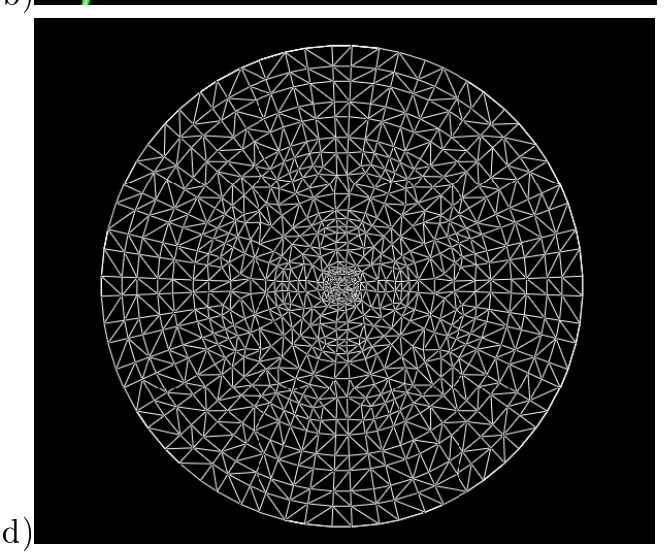

Figure 4. Meshes resulting from direct extrapolation and Delaunay tetrahedrazation of Gauss points.

\section{TENSOR VISUALIZATION}

\subsection{Hedgehogs}

Scientific visualization is to a large extent concerned with the post-processing of results from simulations, where results are typically scalar and vector fields. For example, Figure 5(a) is a visualization of a rotating velocity field using simply arrows for visualization. Drawing of arrows in tangent direction (to the resulting vector field) at each nodal point is one the simplest ways to visualize a vector field in the $2 \mathrm{D}$ plane. When it comes to visualizing principal components 

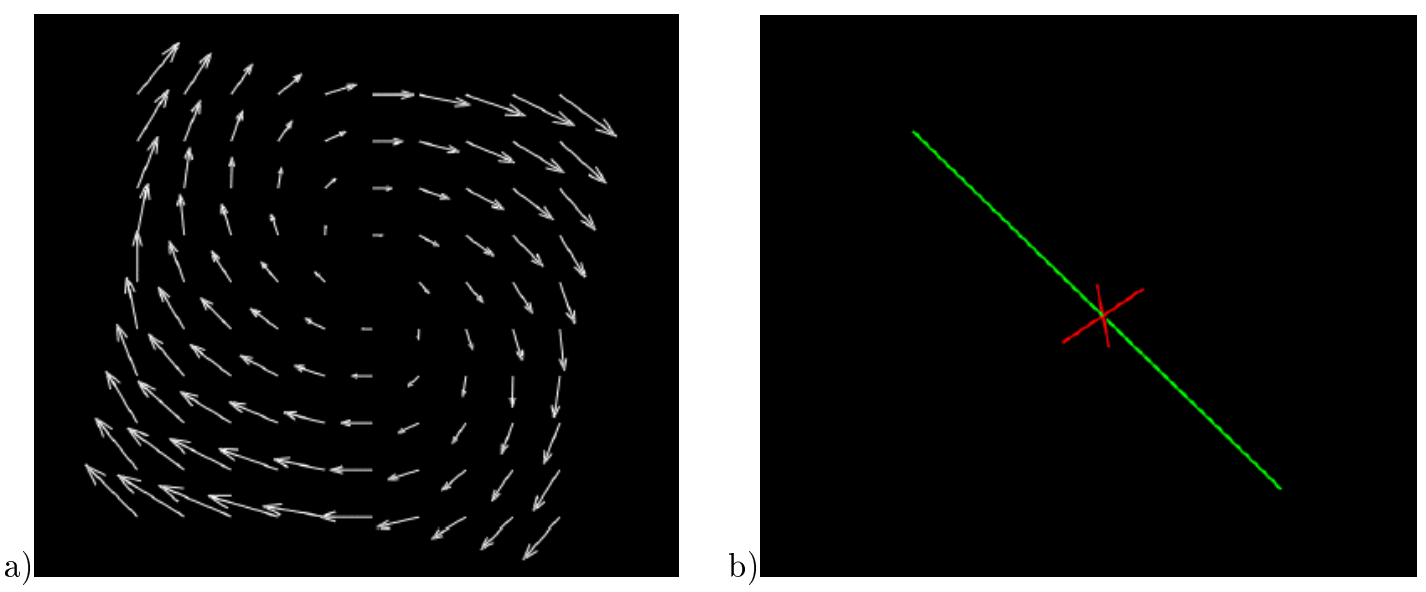

Figure 5. (a) Visualization of a rotating vector field, (b) principal stress hedgehog, color-coded by sign (red is tension, positive, and green is compression, negative); line length represents principal stress magnitude.

of a 3D tensor, similar approaches can be used. Figure 5(b) presents one such approach, using hedgehogs, in which three lines are used to present the three eigenvalues (along corresponding eigenvectors) associated with a single 3D tensor. In this example, we use color for sign (red is tension, positive, and green is compression, negative) and the length of a line to indicated magnitude of the three eigenvalues of the tensor. Even with only one 3D tensor being visualized the relative size of the lines is not quite clear, at least it is not clear by merely looking at a single projection. The orientation of the lines plays a major role in perceiving visually the actual magnitudes of the principal stress values.

Nevertheless, hedgehogs are a simple method to visualize principal stresses. Figure 6 shows stress tensor hedgehogs for our three examples. Figures $6(a, b, c)$ present stress hedgehogs for the single, dual and load couples on a half-space model. Despite a large number of stress hedgehogs, the stress trajectories are evident. The analyst can use such visualizations to gain Copyright (C) 2001 John Wiley \& Sons, Ltd.

Int. J. Numer. Anal. Meth. Geomech. 2001; 01:1-6 Prepared using nagauth.cls 
understanding of the response within a solid. However, the examples shown in Figures $6(\mathrm{a}, \mathrm{b}, \mathrm{c})$ are rather simple and for such simple examples we can intuitively predict orientations and magnitudes of principal stresses.
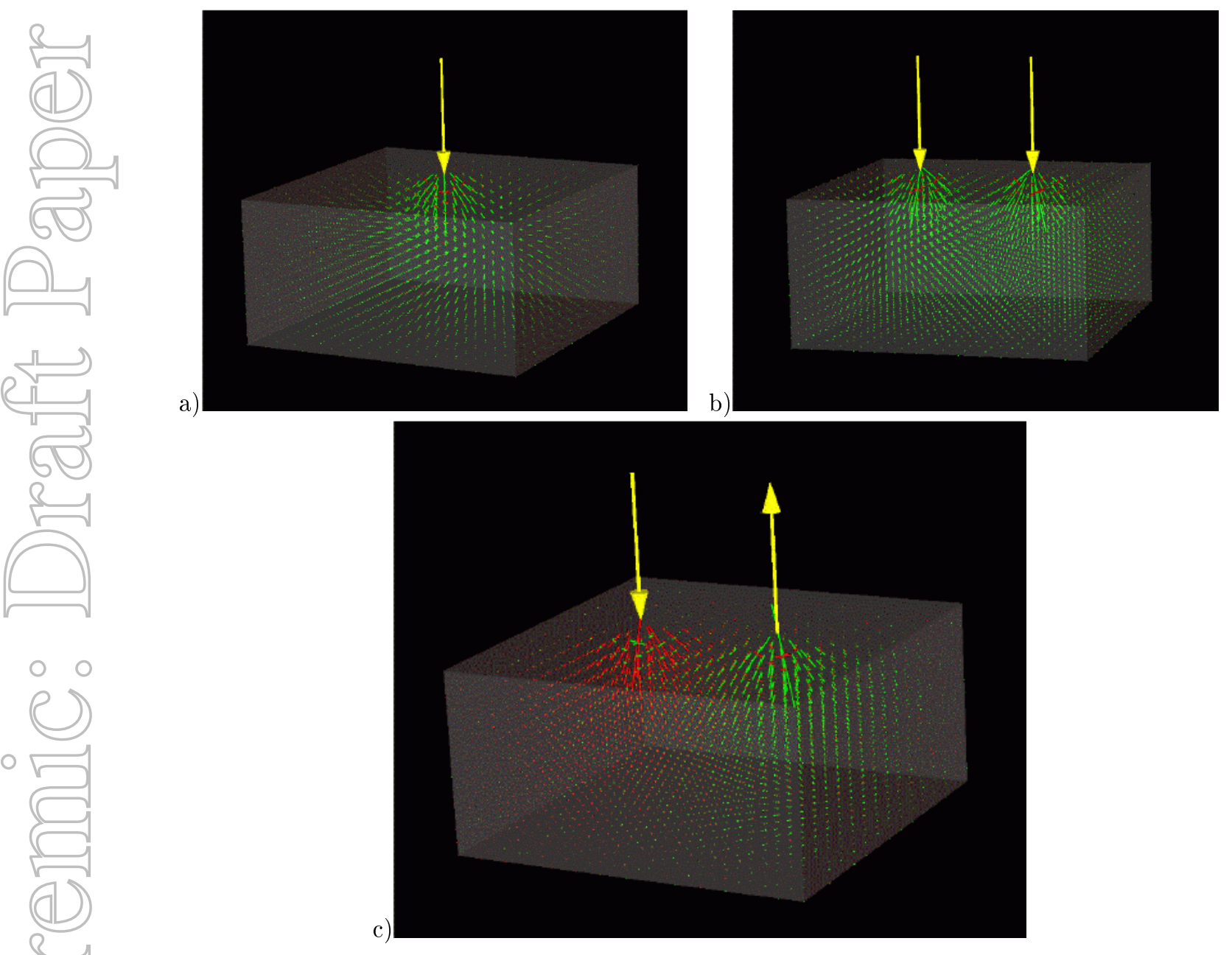

Figure 6. Stress hedgehogs for a single (a), dual (b) and load couples (c) loads on a half space model.

When it comes to visualizing results from numerical geomechanics simulations for realistic objects, hedgehogs are no longer effective. Figures $7(\mathrm{a}, \mathrm{b})$ present principal stress hedgehogs Copyright (C) 2001 John Wiley \& Sons, Ltd. Int. J. Numer. Anal. Meth. Geomech. 2001; 01:1-6 Prepared using nagauth.cls 
for our examples involving pile foundations. Figure $7(\mathrm{a})$ presents a view of principal stress hedgehogs for one layer of the four-pile group close to the surface. The results shown here are taken from one time step of a seismic simulation involving a four-pile group embedded in soil. This presentation of principal stress hedgehogs does not reveal the mechanical behavior of the system. The finite element model used for this four-pile group was rather coarse and, consequently, stress results are not of high quality. With such a deficient stress field resulting from a coarse mesh, the visualization using principal-stress hedgehogs does not convey appropriately the underlying distribution of stresses. Figure 7(b) presents principal
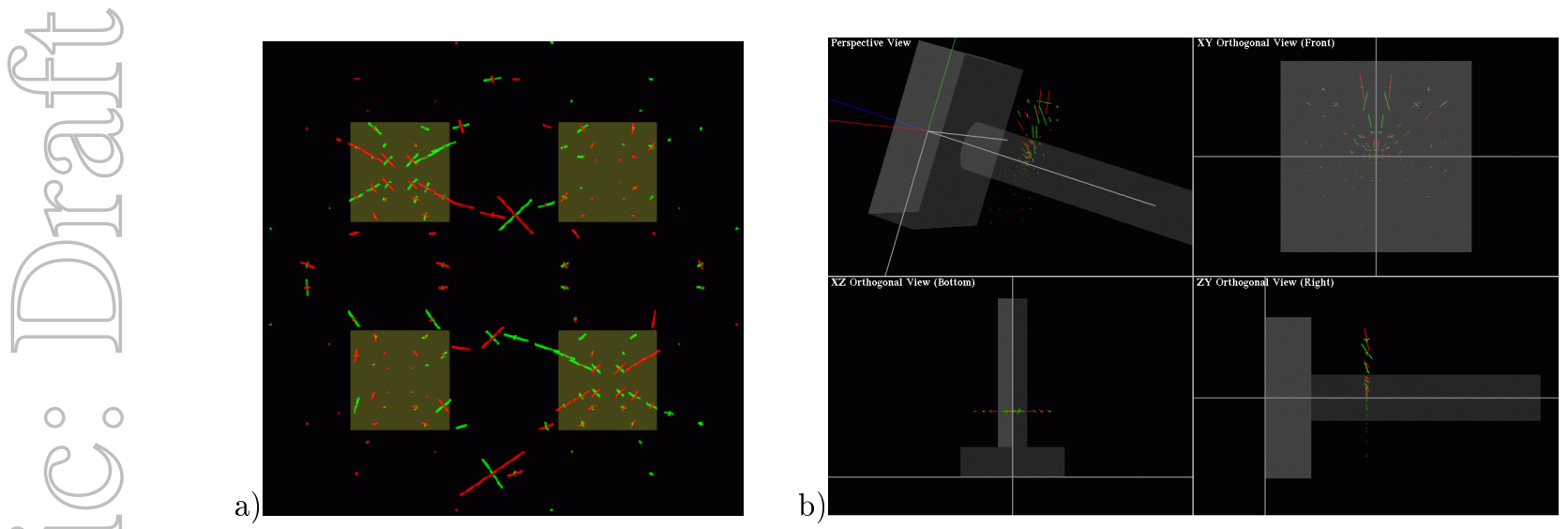

Figure 7. (a) Principal stress hedgehogs for one layer of four-pile group close to the surface, (b) principal stress hedgehogs for a layer just beneath the pile cap for a single pile.

stress hedgehogs for a layer just beneath the pile cap for a single pile. This figure shows a stress field taken from one time step of dynamic simulation. While it is obvious that the four projections (one perspective and three orthogonal views) improve the visualization quite a bit, the stress field is too complicated to be understood using just hedgehogs. 


\subsection{Hyperstreamlines}

A better representation of the stress field in the solid can be obtained by using hyperstreamlines. Dickenson [5] introduced the concept of tensor lines into visualization. These are curves that are everywhere tangent to one of the principal components of the tensor. Delmarcelle and Hesselink [3, 4] extended this idea to hyperstreamlines. A hyperstreamline is a tensor line with a tube or helix structure defined by the other two eigenvalues and eigenvectors.

Figures $8(\mathrm{a}, \mathrm{b}, \mathrm{c})$ show hyperstreamlines for major, intermediate and minor principal stresses for a point-load example. In this case, hyperstreamlines quite effectively describe the stress tensor field. It is particularly interesting to observe the "flows" of the minor principal stress (compressive principal stress) in Figure 8(a) and the major principal stress (tensile principal stress) in Figure 8(c). Color clearly presents the magnitudes of principal stresses. Here, blue depicts high values and red low values. It is also interesting that for the intermediate principal stress tensor hyperstreamlines the color code is alternating cyclically. This is explained by the small variations (due to numerical errors) of the constant principal stress value. The same can be observed for the major principal stress tensor field close to the point-load application point in Figure 8(c).
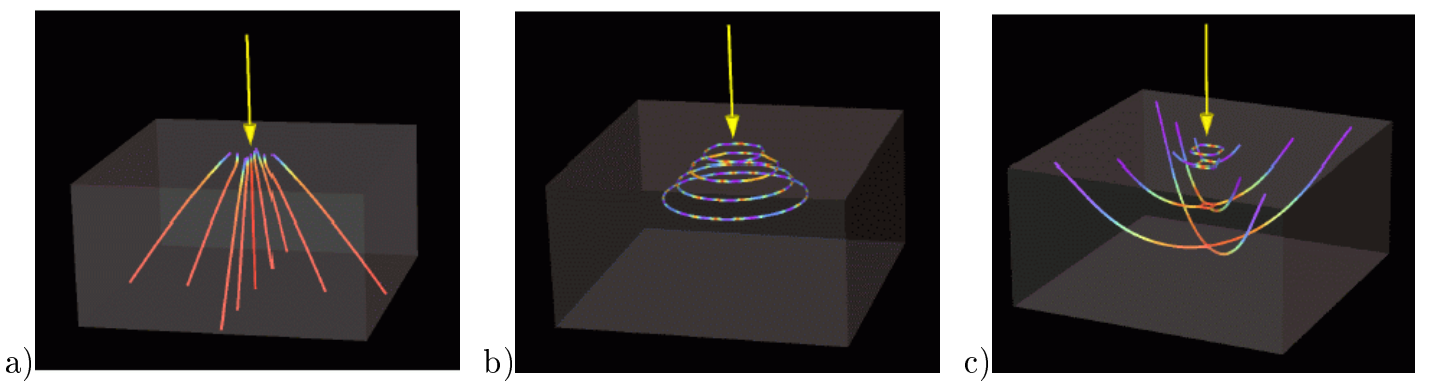

Figure 8. Hyperstreamlines for minor, intermediate and major principal stress for a point-load.

Copyright (C) 2001 John Wiley \& Sons, Ltd.

Int. J. Numer. Anal. Meth. Geomech. 2001; 01:1-6

Prepared using nagauth.cls 
Figures $9(\mathrm{a}, \mathrm{b})$ show hyperstreamlines for the minor principal stress tensor for a two pointload data set and in a load couple data set.
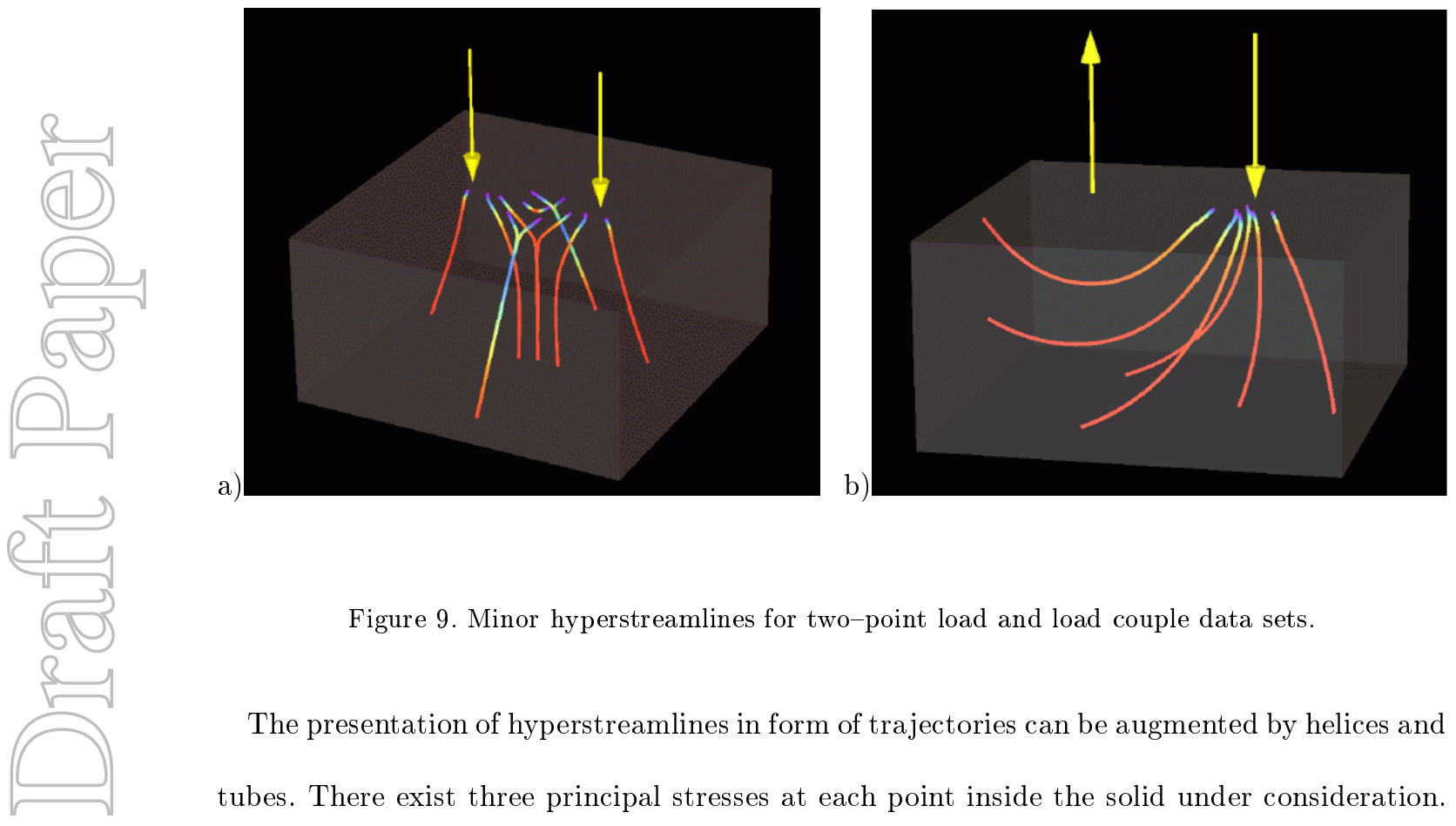

Figure 9. Minor hyperstreamlines for two-point load and load couple data sets.

The presentation of hyperstreamlines in form of trajectories can be augmented by helices and tubes. There exist three principal stresses at each point inside the solid under consideration. The hyperstreamlines present only one of the principal stresses (the minor, intermediate or major one). At each point in space we do have available the two other principal stresses. The addition of helices and tubes serves the purpose of showing all three principal stresses of a tensor on a single visualization. The two orthogonal dimensions of a helix (a cross) or a tube (ellipsoid) are used to represent the two principal stresses while color is used to show the main component of the principal stress along the hyperstreamline. In other words, a helix or tube hyperstreamline is a tensor line with a helix or tube defined by the other two principal components and principal directions of a tensor. Figures $10(\mathrm{a}, \mathrm{b})$ shows the variations of hyperstreamlines presentation for a single point load example: (a) hyperstreamlines drawn as helix just beneath the point load, (b) hyperstreamlines drawn as tube just beneath the Copyright (C) 2001 John Wiley \& Sons, Ltd.

Int. J. Numer. Anal. Meth. Geomech. 2001; 01:1-6 Prepared using nagauth.cls 
point load. For the single point load, the five helix and tube hyperstreamlines are shown in Figures 10(a,b) respectively.
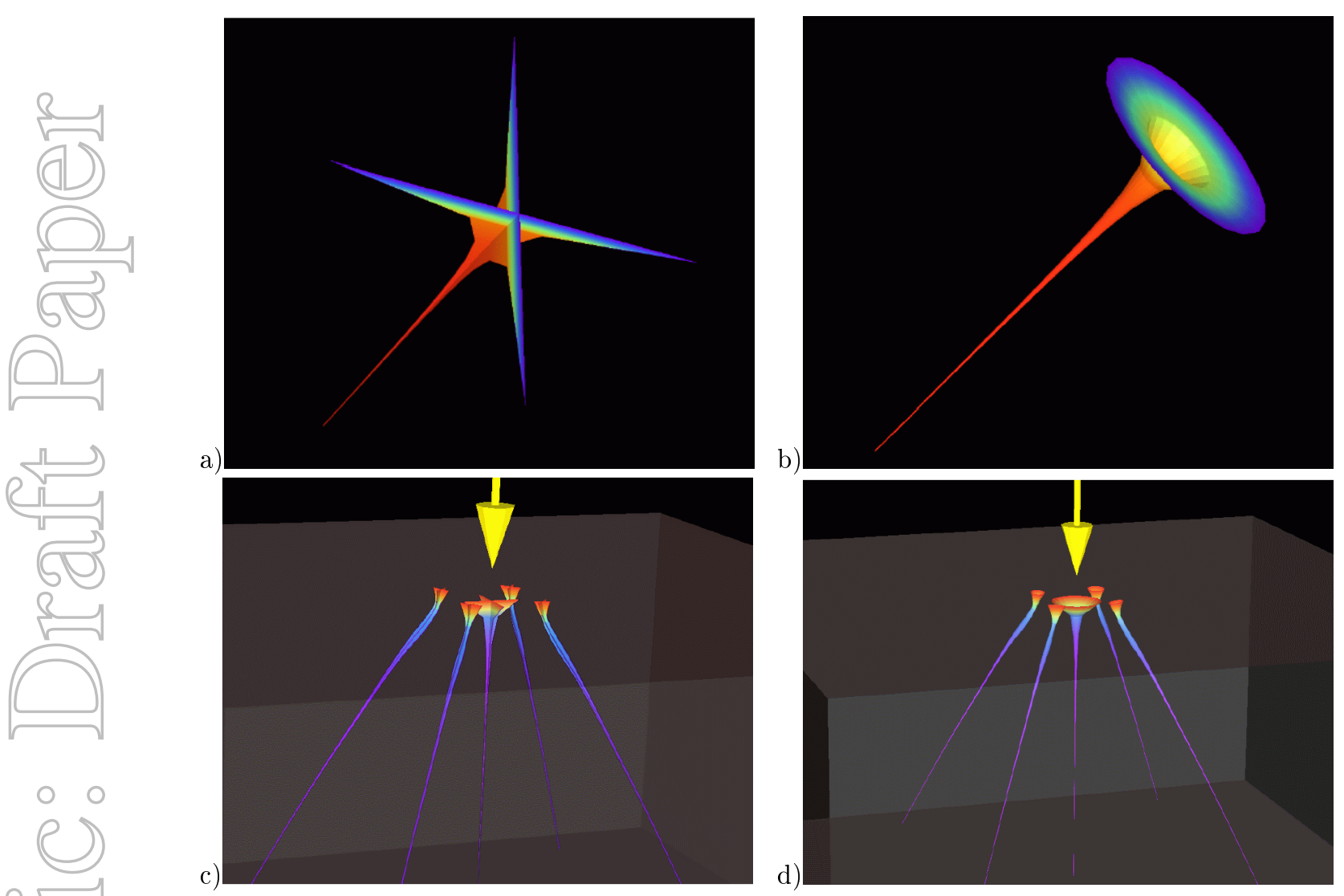

0

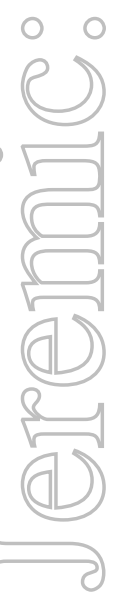

Figure 10. Helix $(\mathrm{a}, \mathrm{c})$ and tube $(\mathrm{b}, \mathrm{d})$ hyperstreamlines for a single-point load example.

Figures 11(a,b) show tube hyperstreamlines for the minor principal stress for two point load and the couple. It is interesting to note that the tube hyperstreamlines perform quite nicely if all the principal stress values are of the same sign. If, on the other hand, one of the principal stresses changes sign, the tube hyperstreamline will cease to exist. In this case, the ellipsoid part of the tube hyperstreamline will be converted to a paraboloid. Before that Copyright (C) 2001 John Wiley \& Sons, Ltd.

Int. J. Numer. Anal. Meth. Geomech. 2001; 01:1-6 Prepared using nagauth.cls 
happens, the tube hyperstreamline will, when one of the two other principal stresses becomes zero, degenerate. This behavior is shown in Figures 11(a,b).
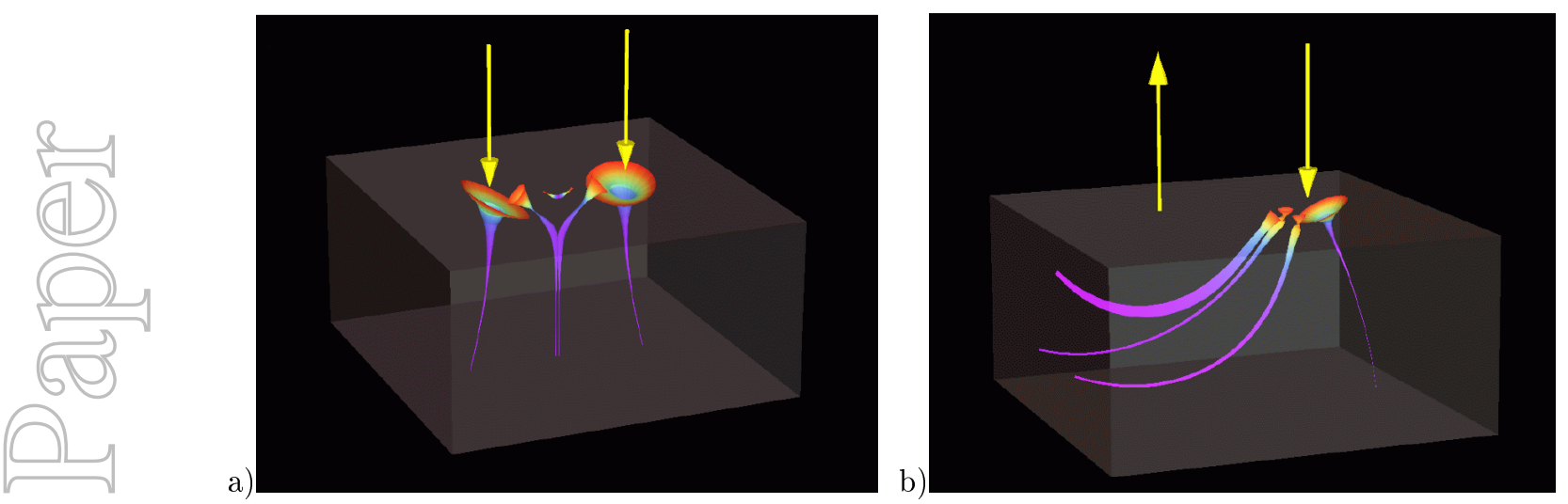

Figure 11. Tube hyperstreamlines for minor principal stress for (a) two-point loads and (b) load couple examples.

Figure 12 shows helix hyperstreamlines for the intermediate principal stress for a two-point load and a load couple examples. Considering Figure 12(a), the two-point load example, the variation of the intermediate principal stress is presented using color along the hyperstreamline. The variation of the other two components of the principal stress, given by the helix dimensions at the specific point, shows that one of the values (minor principal stress) has a finite value while the other one (major principal stress) is close to zero. When comparing Figures 12(a) and 6(b), it becomes obvious that the major principal stress components are small or close to zero. In some instances they even become negative. As for the tube hyperstreamlines, negative values for one (or both) of the other principal stress values makes this visualization approach unusable.

Figure 13 shows hyperstreamlines for the single-pile data set. The first three figures Figures 13(a,b,c), show the minor principal stress hyperstreamlines in terms of (a) line Copyright (C) 2001 John Wiley \& Sons, Ltd.

Int. J. Numer. Anal. Meth. Geomech. 2001; 01:1-6 Prepared using nagauth.cls 

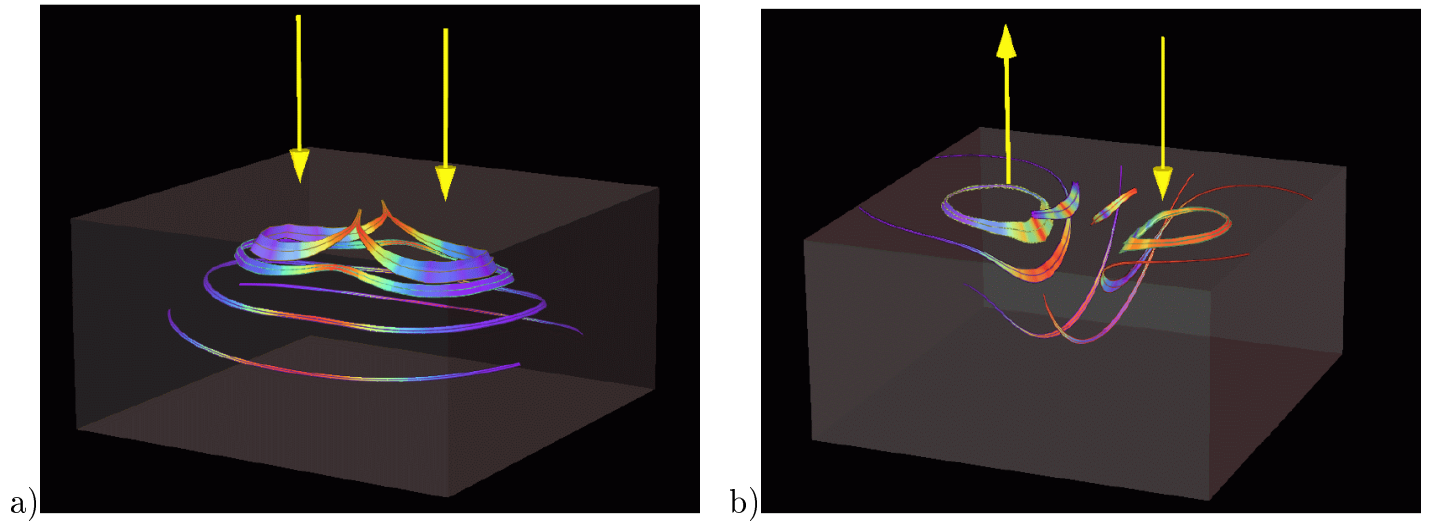

Figure 12. Helix hyperstreamlines for the intermediate principal stress for (a) two-point load and the (b) load couple examples.

hyperstreamlines, (b) tube hyperstreamlines, and (c) helix hyperstreamlines. The starting point for these hyperstreamlines is just outside the pile, in the soil, and the end points are on the other side of the pile, again in the soil. Figure 13(d) shows major principal stress hyperstreamlines starting in soil, extending through the solid pile, and ending in the soil on the other side.

There are a number of details worth mentioning. In Figures 13(a,b,c), minor principal stress hyperstreamlines are shown, which means that we follow the compressive stress in tangent direction and then, in the case of helix and tube hyperstreamlines, plot the other two principal stresses. We consider the shape of hyperstreamlines: The upper four hyperstreamlines are "bending upward" while the lower ones are mainly horizontal. This means that, in the upper part of the pile, compressive stresses are inclined (almost vertical), which is expected for the bending deformation of a pile. Beginning at some depth, however, the pile is not subjected to bending any longer. The horizontal traces of minor principal stresses at the left (front) side in the upper portion of the pile are also expected, as this side should have large tensile stresses. Copyright (C) 2001 John Wiley \& Sons, Ltd.

Int. J. Numer. Anal. Meth. Geomech. 2001; 01:1-6 Prepared using nagauth.cls 

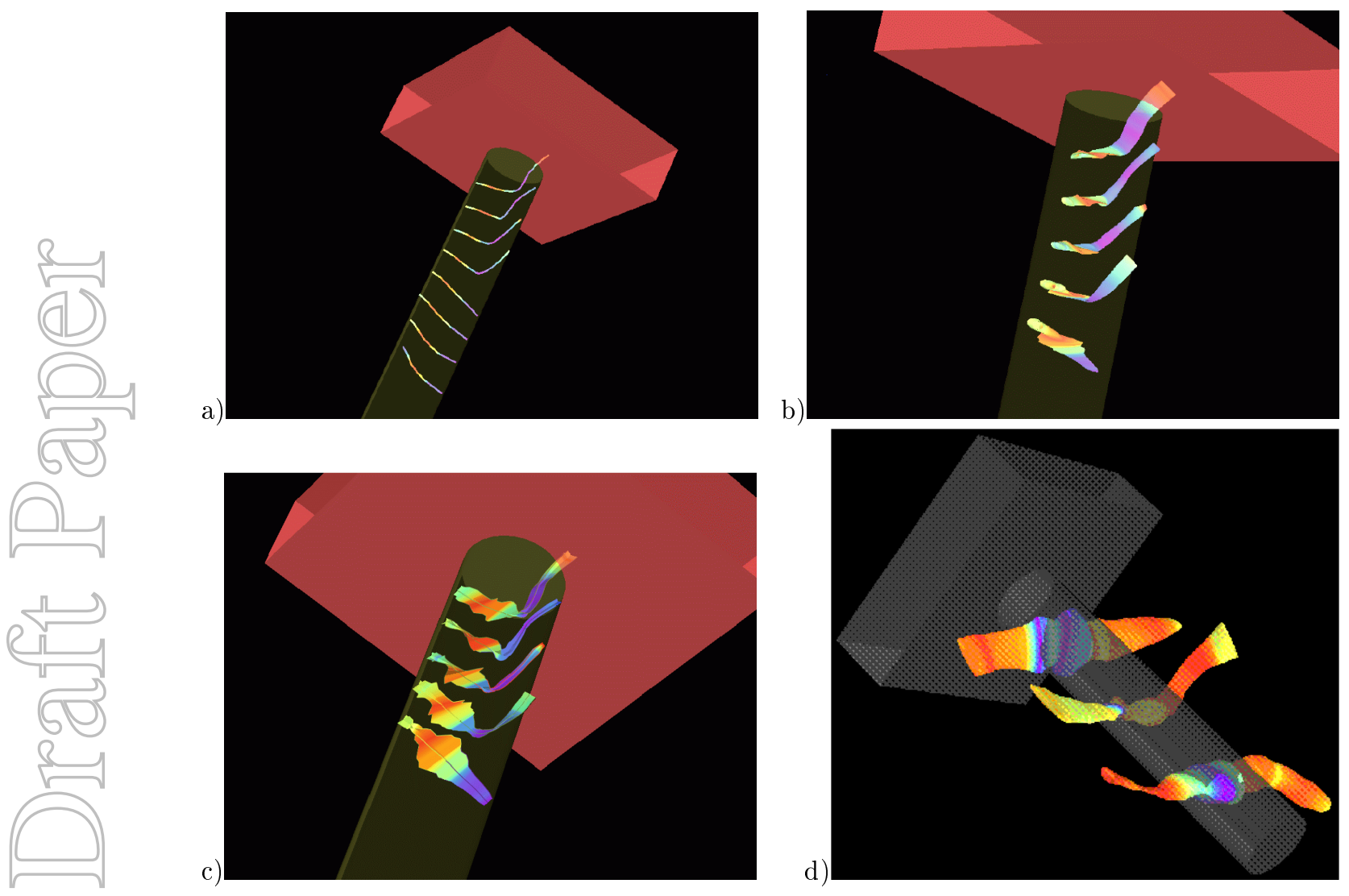

Figure 13. Minor principal stress hyperstreamlines for one-pile model: (a) hyperstreamlines; (b) tube hyperstreamlines, (c) helix hyperstreamlines; and (d) major hyperstreamlines extending through the soil and concrete.

Those tensile stresses are best seen in helix hyperstreamlines in Figure 13(c).

Figure 13(d) shows the change of the major principal stress (tensile) along its own trajectory. We added tubes representing the other two principal stresses (intermediate and minor). First, a significant variation is observed for the major principal stress (depicted in changing color) as it enters and leaves the region occupied by the concrete pile. Second, significant rotation of the principal stress triad is observed, particularly in the uppermost tube hyperstreamline, Copyright (C) 2001 John Wiley \& Sons, Ltd.

Int. J. Numer. Anal. Meth. Geomech. 2001; 01:1-6 Prepared using nagauth.cls 
which indicates a quite different stress state (magnitude and orientation) in the concrete pile and the surrounding soil. Third, the variation of stress values is much smaller in the soil than in concrete, and the variations become smaller the deeper a hyperstreamline is.

\subsection{Hyperstreamsurfaces}

Despite the fact that we can generate powerful visualizations with hyperstreamlines, this method can only show stress lines that follow the tangent of one of the principal stresses. Therefore, visualization results are highly dependent on the initial point where we start to follow the tangent to one of the principal stresses. By increasing the number of hyperstreamlines one can gain a better understanding of overall structure of the tensor space but this approach has its limitations. Figures 14 shows visualizations based on relatively large number of hyperstreamlines. Figures 14(a,b,c) show minor principal stress for a double-load data set with (a) nine, (b) sixteen, and (c) twenty five hyperstreamlines. Figures $15(\mathrm{~d}, \mathrm{e}, \mathrm{f})$ show minor principal stress for a load couple data set with (a) nine, (b) sixteen, and (c) twenty five hyperstreamlines.

This concept of hyperstreamlines can be extended to so-called hyperstreamsurfaces. The hyperstreamsurface is a visualization that uses a set of points on an open (or closed) curve to construct a number of hyperstreamlines, which are then connected using polygons. Figure 15 shows hyperstreamsurfaces for a point-load data set. Figures 15(a,b,c) show hyperstreamsurfaces for the minor, intermediate, and major principal stresses. Figures 16(a,b,c,d,e,f) show hyperstreamsurfaces for the minor, intermediate, and major principal stresses, for the double-load and load-couple data sets.

Figures 17(a,b) shows hyperstreamsurfaces for minor principal stress (compression) for (a) Copyright (C) 2001 John Wiley \& Sons, Ltd.

Int. J. Numer. Anal. Meth. Geomech. 2001; 01:1-6 Prepared using nagauth.cls 

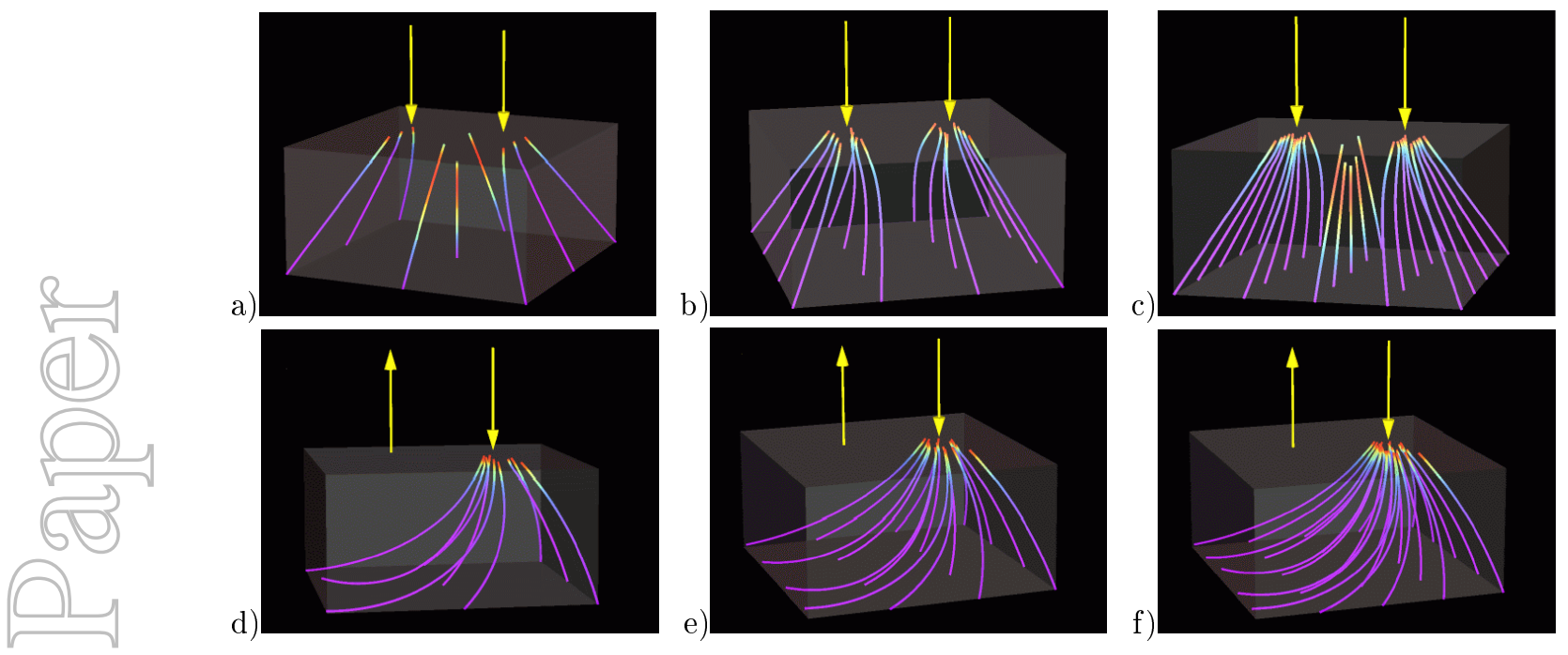

Figure 14. Families of hyperstreamlines representing minor principal stresses for the double-point and the load-couple data set.
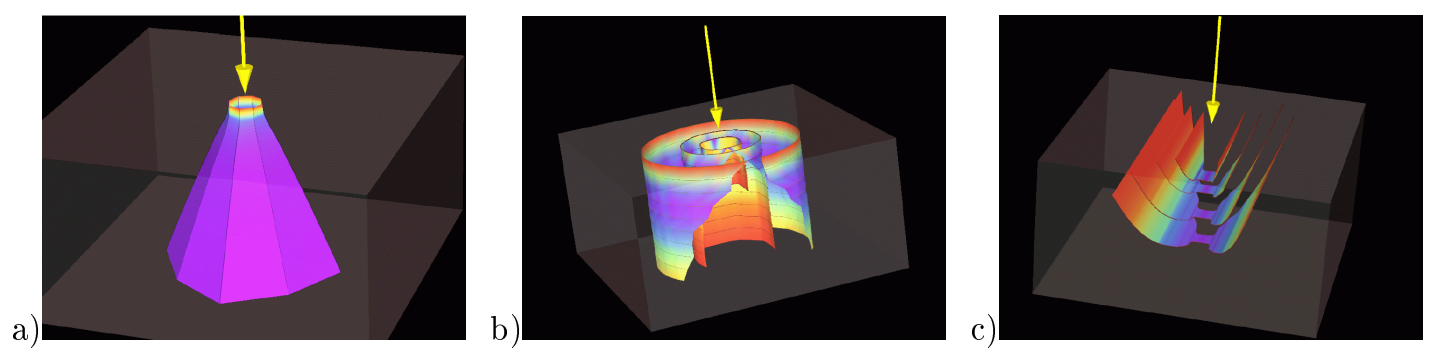

Figure 15. Hyperstreamsurfaces for (a) minor, (b) intermediate, and (c) major principal stresses for point-load data set.

a single-pile and (b) a four-pile group example. In Figure 17(a), the hyperstreamsurface starts at the center line of a pile and extends through the concrete (in both directions) until it reaches surrounding soil. Figure 17(a) exhibits the same behavior as Figure 13(a). Both figures represent the minor principal stress for the same single-pile simulation. The hyperstreamsurface shown in Figure 17(a) reveals the stress field in more details. We notice Copyright (C) 2001 John Wiley \& Sons, Ltd. Int. J. Numer. Anal. Meth. Geomech. 2001; 01:1-6 Prepared using nagauth.cls 

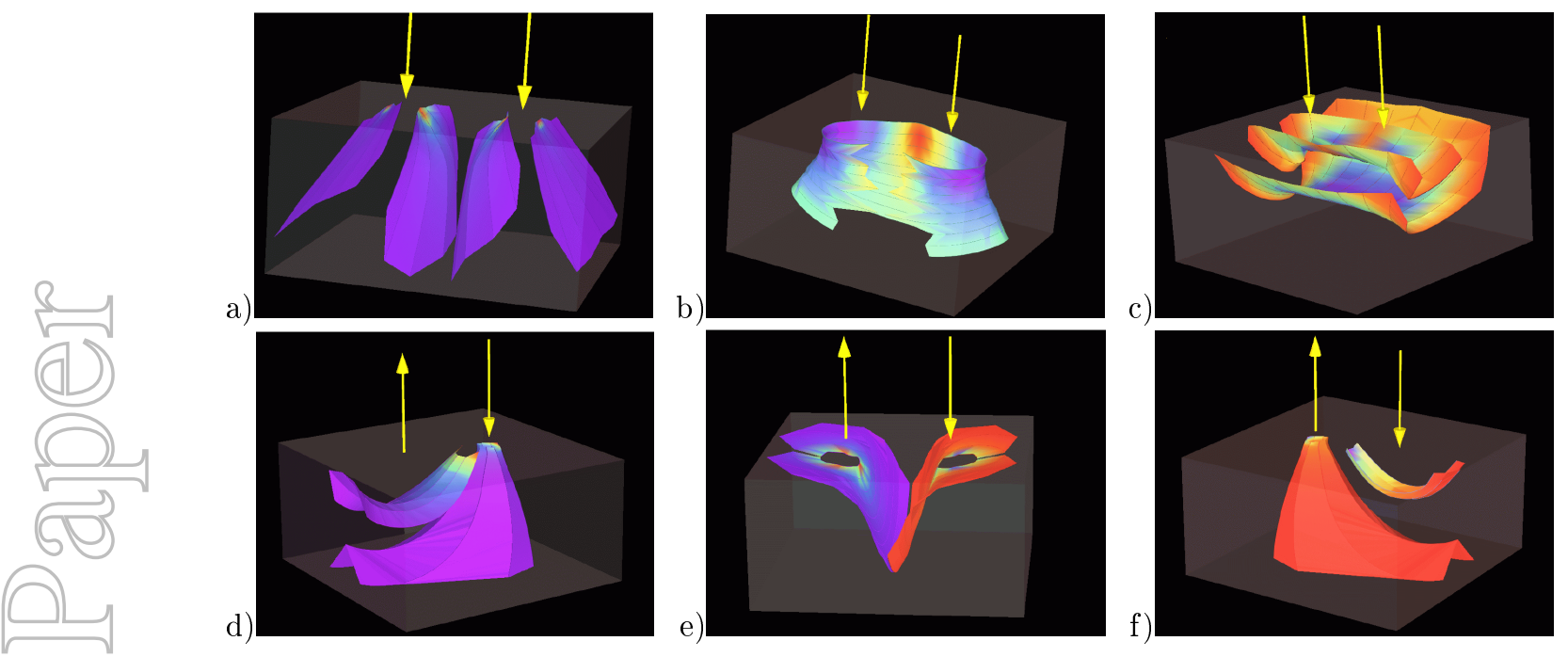

Figure 16. Hyperstreamsurfaces for (a,d) minor, (b,e) intermediate, and (c,f) major principal stresses (for the double-load $(a, b, c)$ and load-couple (d,e,f) data set).

the twisting and "discoloring" of the hyperstreamsurface toward the bottom of the pile. This can be explained by the fact that the stress field in the concrete pile is far away from the main bending effects close to the surface. The state of stress thus deviates just by a small amount from the initial state induced by the self weight. Such small change in stresses leads to a near-uniform green hue coloring. A "kink" in direction and color is noticed close to the surface. This is a part of the hyperstreamsurface that ends in the soil and thus, by moving from stiff (concrete) toward soft (soil) medium, has much smaller minor (compressive) stresses. The reason for such behavior can be deduced from Figure 13(a), showing hyperstreamlines: The minor principal stress is bend upward (due to large compressive stresses resulting from bending), and the stress path extends into the concrete pile cap. If more hyperstreamlines were used in constructing a hyperstreamsurface, smoother transition from soil interface to concrete cap would result. However, with the current choice, there is a region inside the Copyright (C) 2001 John Wiley \& Sons, Ltd. Int. J. Numer. Anal. Meth. Geomech. 2001; 01:1-6 Prepared using nagauth.cls 
pile and the adjacent soil, toward the upper-end of a pile, that is not properly covered by hyperstreamlines/hyperstreamsurfaces.
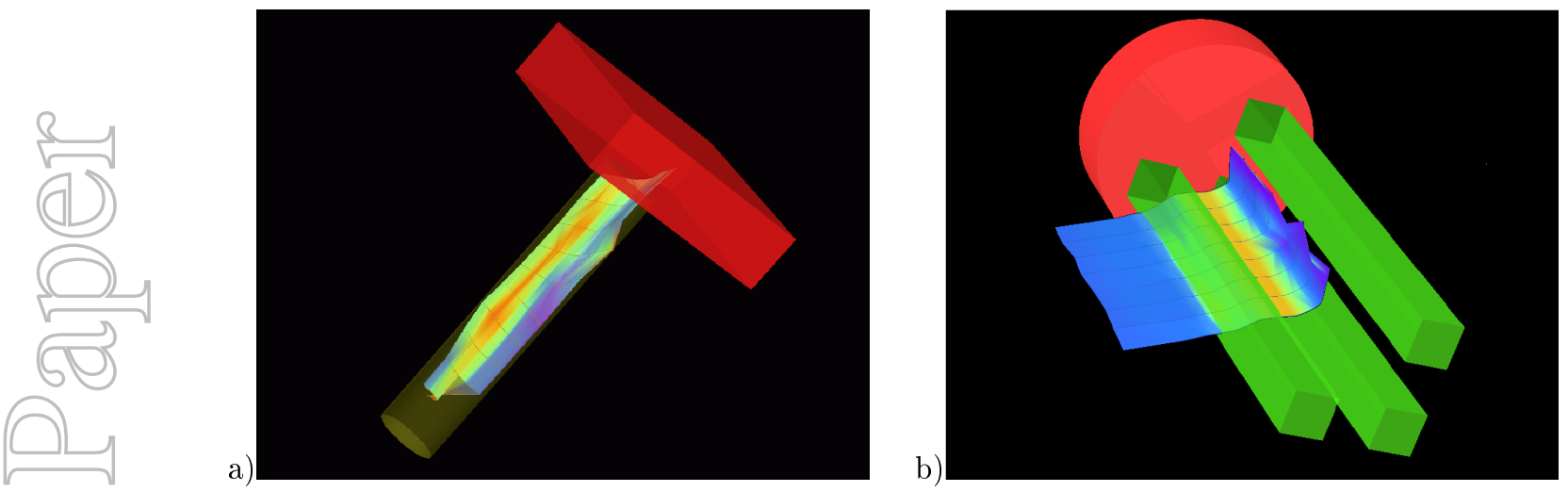

Figure 17. Hyperstreamsurfaces for minor principal stress (compression) for (a) a single-pile and (b) a four-pile group.

Figure 17(b) represents the minor (compressive) principal stress hyperstreamsurface for the four-pile group. In this figure, we have removed one pile from the model to simplify the view. An interesting effect is "shading," apparent behind the left pile in this figure. The hyperstreamsurface starts, at the center of that pile and extends outside, ending in soil. This pile group is much stiffer than the one-pile example. The effects of bending are much smaller, and the four-pile group behaves like a stiff frame embedded in soil. The observed deformation pattern is thus closer to horizontal translation with a large horizontal resistance and small bending. The hyperstreamsurface extends into the soil outside the pile group, with apparent compressive stress. However, just behind the pile, but inside the pile group, the minor principal stress changes significantly in value and then curves by almost $90^{\circ}$. The twist of the minor principal stress hyperstreamsurface clearly demonstrates the pile-group effect: Maximal compressive stress (minor principal stress) is acting between two piles in direction Copyright (C) 2001 John Wiley \& Sons, Ltd.

Int. J. Numer. Anal. Meth. Geomech. 2001; 01:1-6 Prepared using nagauth.cls 
perpendicular to the pile loading direction. This findings is contrary to common belief about the state of stresses in pile groups. More important, a large "shading" effect is observed, which, when combined with the twisting of the minor principal stress, leads us to conclude that the two back piles are mostly loaded in direction perpendicular to the global loading direction.

\subsection{Isosurfaces}

In order to gain better understanding of the state of stress in a solid, it is interesting to determine the positions where one or two of the principal stresses change sign. Figures 18(a,b) show an isosurface close to the pile foundation with one positive principal stress value, Figure 18(a), and with two positive principal stress values, Figures 18(b). Figure 18(a) clearly shows
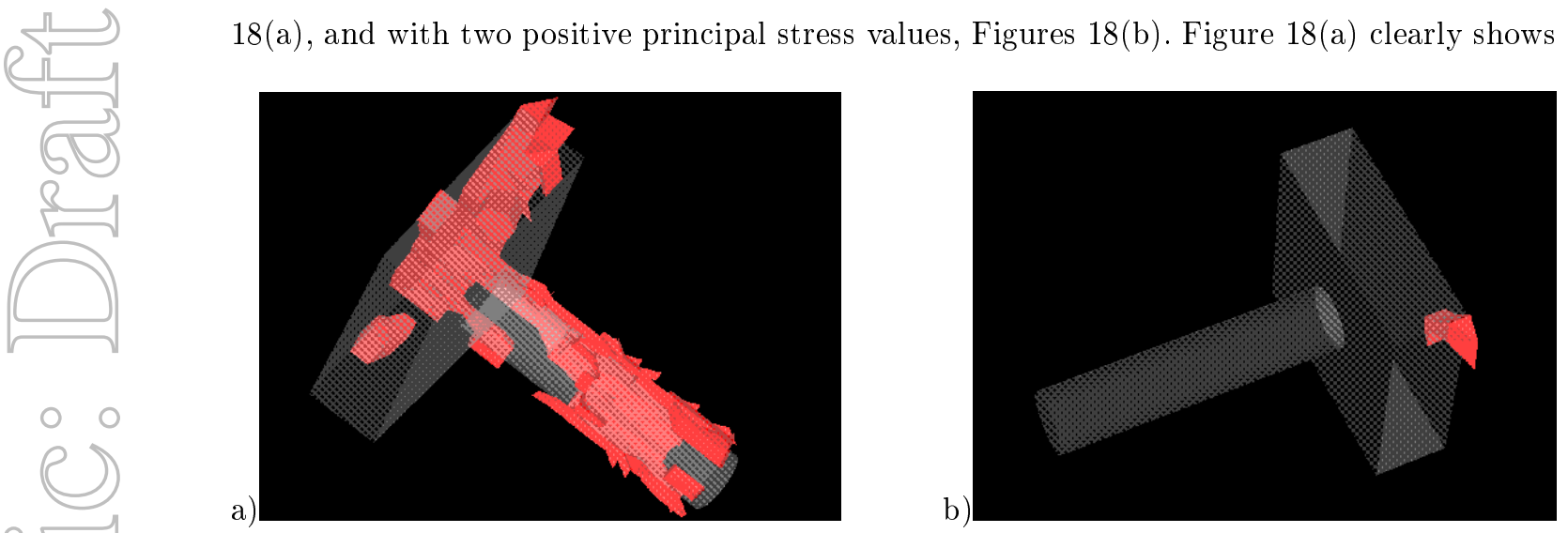

Figure 18. Isosurface of regions with (a) one positive principal stress value and (b) two positive principal stress values.

the zones with some tensile stresses. These zones are mostly concentrated near the concretesoil interface as expected and represent gap openings. The location of these zones does not follow the concrete-soil interface exactly, most likely due to numerical errors produced by the algorithm used for interpolating stresses. It is also interesting that a small region where two principal stresses are positive, at the corner of the top of pile cap, becomes evident. 


\subsection{Time-dependent Tensor Fields}

The visualization methods we have described in the previous sections can be extended to dynamic events, to time-dependent simulated data. Unfortunately, we cannot present visualization animation of the dynamic data in a paper. However, we will present image sequences for dynamic data. It is our experience that very good results can be obtained by animating hyperstreamlines and hyperstreamsurfaces.

Figure 19 presents a sequence of hyperstreamline visualizations of the single-pile dataset. This series of snapshots is for time steps 147-161 of a dynamic simulation using Kobe earthquake input motions. The motion of two minor principal stress hyperstreamlines is shown. It seems that the animated visualization is not very; it requires experience to understand the changes in the stress field.

Figure 20 presents a sequence of hyperstreamsurfaces for the same simulation. This animation conveys the changes of the minor principal stress state in the pile and surrounding soil much better than using plain hyperstreamlines for animation. It can be seen that the minor principal stress follows cycles of compression. We can only observe cycles of compression while cycles of tension (in the concrete pile) would have to be shown using a second hyperstreamsurface for the major principal stress.

Copyright (C) 2001 John Wiley \& Sons, Ltd.

Int. J. Numer. Anal. Meth. Geomech. 2001; 01:1-6 Prepared using nagauth.cls 

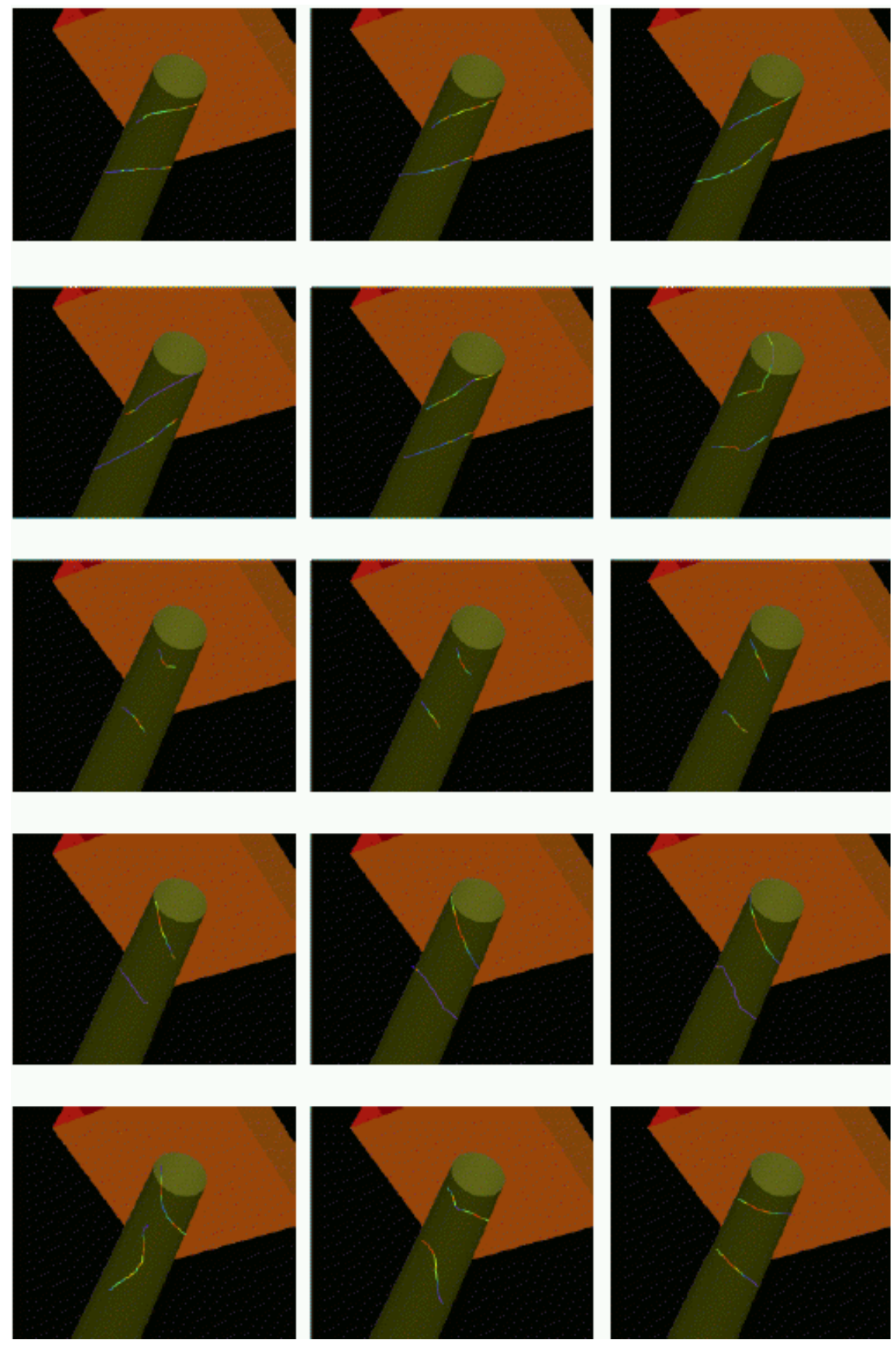

20

○

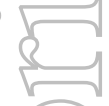

Figure 19. Sequence of snapshots for two minor principal stress hyperstreamlines for 15 time steps of a dynamic simulation using Kobe earthquake input motions. 

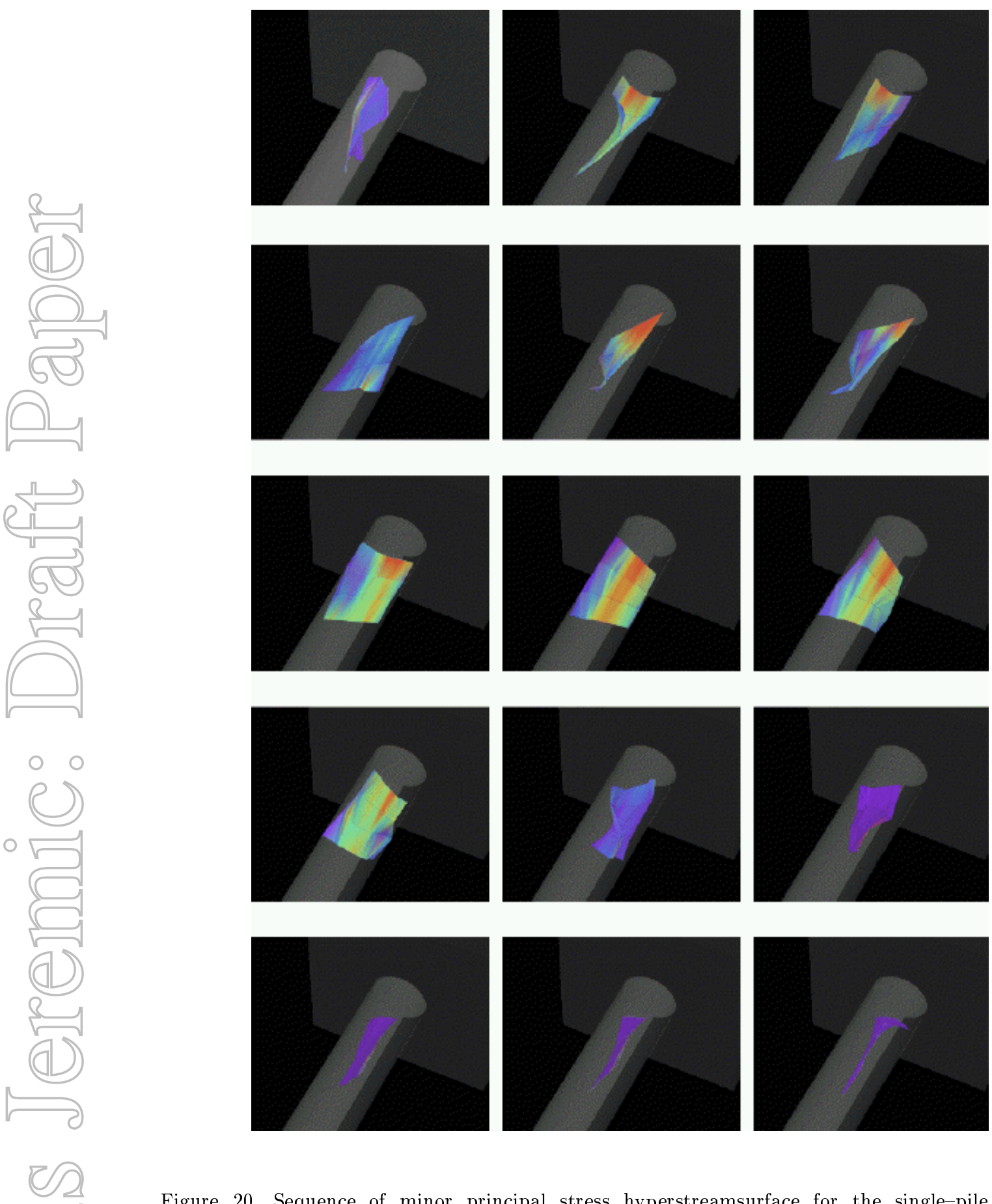

0

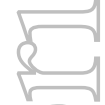

Figure 20. Sequence of minor principal stress hyperstreamsurface for the single-pile dynamic simulation, 15 time steps shown.

Copyright (C) 2001 John Wiley \& Sons, Ltd.

Int. J. Numer. Anal. Meth. Geomech. 2001; 01:1-6

Prepared using nagauth.cls 


\section{CONCLUSION}

We have discussed different approaches to visualizing tensors for computational geomechanics. We have focused on the visualization of the stress tensor and have presented visualization of the principal stress tensor using hedgehogs, hyperstreamlines, hyperstreamsurface, and isosurfaces. We have also presented extensions of some of these methods for the visualization of dynamic data, using the behavior of piles during an earthquake event as examples.

We have provided visualizations for simple and complicated boundary value problems. A number of interesting findings could be made using the presented visualization methods especially for the analysis of single-pile and pile-group behavior.

\section{ACKNOWLEDGEMENT}

This work was supported through a number of grants and contracts. We received support through:

The National Science Foundation under contract EEC-9701568 to the Pacific Earthquake Engineering Research Center, ACI 9624034 (CAREER Award), through the Large Scientific and Software Data Set Visualization (LSSDSV) program under contract ACI 9982251, and through the National Partnership for Advanced Computational Infrastructure (NPACI); the Office of Naval Research under contract N00014-97-1-0222; the Army Research Office under contract ARO 36598MA-RIP; the NASA Ames Research Center through an NRA award under contract NAG2-1216; the Lawrence Livermore National Laboratory under ASCI ASAP Level-2 Memorandum Agreement B347878 and under Memorandum Agreement B503159; and the North Atlantic Treaty Organization (NATO) under contract CRG.971628 awarded to the University of California, Davis. We also acknowledge the support of ALSTOM Schilling Robotics and SGI. We thank the members of the Visualization and Graphics Research Group at the Center for Image Processing and Integrated Computing (CIPIC) at the University of California, Davis. In addition, we thank the members of the Computer Graphics Group at the University of Kaiserslautern, Germany, especially Tom Bobach, 
Christoph Garth, Aragorn Rockstroh, Xavier Tricoche, Thomas Wischgoll, and Wang Yi for their programming efforts and valuable discussions.

\section{REFERENCES}

1. Hensheng Bao, Jacobo Bielak, Omar Ghattas, Loukas F. Kallivokas, David R. O'Hallaron, Jonathan Richard Shewchuk, and Jifeng Xu. Earthquake ground motion modeling on parallel computers. In Supercomputing '96, 1996.

2. Hesheng Bao, Jacobo Bielak, Omar Ghattas, Loukas F. Kallivokas, David R. O'Hallaron, Jonathan R. Shewchuk, and Jifeng Xu. Large-scale simulation of elastic wave propagation in heterogeneous media on parallel computers. Computer Methods in Applied Mechanics and Engineering, 152(1-2):85-102, January 1998.

3. Thierry Delmarcelle and Lambertus Hesselink. Visualization of second-order tensor fileds and matrix data. In Arie E. Kaufman and Gregory M. Nielson, editors, Proceedings IEEE Visualization '92,, pages 316-323. IEEE Computing Society Press, Los Alamitos, CA, 1992.

4. Thierry Delmarcelle and Lambertus Hesselink. Visualizing sewcond-order tensor fields with hyperstreamlines. IEEE Computer Graphics \& Applications, 13(4):25-33, 1993.

5. R. R. Dickenson. A unified approach to the design of visualization software for the analysis of field problems. In SPIE Proceedings, volume 1083, Bellingham, Washington, 1989. SPIE - The International Society for Optical Engineering.

6. Fakespace, Inc. Immersive WorkBench. http://www. fakes cape.com

7. Albert Edward Green and Wolfgang Zerna. Theoretical Elasticity. Dover Publications, Inc., 1992.

8. Hans Hagen, Gregory M. Nielson, and Heinrich Müller. Scientific Vizualization, chapter 20.3. IEEE Computer Society Press, 1997.

9. Keith Hjelmstad. Fundamentals of Structural Mechanics. Prentice-Hall, 1997. ISBN 0-13-485236-2.

10. Boris Jeremić, Zhaohui Yang, and Tiejun Li. Large scale, 3D finite element analysis of dynamic soilfoundation-structure interaction. In John Tassoulas, editor, Proceedings of 13th Engineering Mechanics Conference: CD-ROM, page 6 pages, Austin, Texas, May 2000. Engineering Mechanics Division of the American Society of Civil Engineers.

11. Natarajan Sukumar and Mark Rashid. Finite element procedures in applied mechanics, April 2001. ECI212A, Lecture Notes, UC Davis.

Copyright (C) 2001 John Wiley \& Sons, Ltd.

Int. J. Numer. Anal. Meth. Geomech. 2001; 01:1-6

Prepared using nagauth.cls 
12. Olgierd Cecil Zienkiewicz and Robert L. Taylor. The Finite Element Method, volume 1. McGraw - Hill Book Company, fourth edition, 1991.
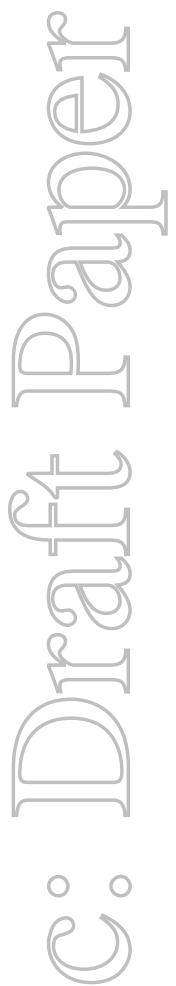

0
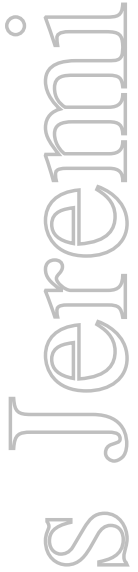

0

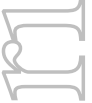

